\title{
Bewuchsuntersuchungen auf Natursteinsubstraten im Gezeitenbereich des Nordsylter Wattenmeeres
}

\author{
G. LUTHER \\ Biologische Anstalt Helgoland (Litoralstation); \\ List/Sylt, Bundesrepublik Deutschland
}

\begin{abstract}
Fowling studies on natural-stone substrates in the tidal zone of the North Sylt wadden sea. Six different types of test substrates were exposed in the tidal zone of the wadden sea near the harbour of List (island of Sylt, North Sea): chalk of Solnhofen, limestone, mottled sandstone, granite, basalt and basalt-lava. The test substrates were fixed to a pannel at the midtide-high-water-level, the midtide-low-water-level and $75 \mathrm{~cm}$ below the latter (sublittoral level). Abiotic and biotic environmental factors decisively affected the growth of settling marine plants and animals. Variations in temperature and salinity as well as in the abundance of planktonic organisms, competitors and predators, caused shiftings in the annual beginning of settlement and in the abundance of settled organisms. Beginning with intense frost in March, the annual dynamics of 1971 were characterised by pronounced oscillations in temperature and salinity. The plankton density was poor in spring. When the sea water suddenly warmed in May, some meroplanktonic larvae appeared in high members. This phenomenon was followed by an intensive settlement on the substrate surfaces. In contrast to 1971, water temperatures remained much more stable in 1972. In this year, holoplanktonic copepods predominated throughout, and settlement was less intensive.
\end{abstract}

\section{EINLEITUNG}

Zwischen dem Substrat und dem Bewuchs durch marine Organismen bestehen enge Wechselbeziehungen. Die Ursachen dieser Wechselbeziehungen beruhen unter anderem auf den physiko-chemischen Eigenschaften der Substrate, die wiederum durch klimatische und hydrographische Umweltfaktoren beeinflußt werden. Experimentelle Studien der Bewuchs-Sukzessionen sind vielfach durchgeführt worden (vgl. EUROPEAN Productivity Agency, 1961), wobei als Substrate zumeist Platten aus Kunststoff, Glas, Zement und Eisen, seltener solche aus gewachsenen Stoffen wie Holz und Schiefer dienten.

In der vorliegenden Arbeit wird ausschließlich der Bewuchs auf natürlichen Gesteinssubstraten untersucht. Die bei Helgoland auftretenden Sedimente (Buntsandsteine und Kalksandsteine) sowie die bei Küstenschutzanlagen verwerteten Materialien (Granite und Basalte) schienen im Hinblick auf Farbe, Härte, Porosität, Textur und chemische Eigenart für die Experimente besonders geeignet. Künstlich hergestellte Platten aus Quarzsand dienten als Kontrollen, um festzustellen, wie weit verschiedene Korngrößen den Bewuchs beeinflussen. 
In-situ-Beobachtungen über den Bewuchs natürlich gewachsener Substrate durch marine Organismen wurden bisher nur im Rahmen ökologischer Studien erwähnt. So berichteten zum Beispiel Nifnburg (1925) über die Besiedlung des Helgoländer Buntsandsteins durch Meeresalgen und BOALCH (1957), CrIsp \& SOUTHWard (1958) über pflanzlichen und tierischen Bewuchs auf Kalkgesteinen der englischen Küste. DоTY (1967), LAwson \& NoRTON (1971) und Munda (1972) beschrieben die Algenbesiedlung auf alten und jungen Eruptivgesteinen, DeN HARTog (1959) und NiEnHuis (1969) auf den Küstenschutzanlagen Hollands. Experimentelle Studien über Bewuchs in Relation zur Härte diverser Naturgesteine führte STEPHENSON (1961) durch.

Im Gegensatz zu den angeführten Veröffentlichungen, deren Beobachtungen sich auf mehr oder weniger verwitterte Substrate beziehen, wurde zu den vorliegenden Versuchen jeweils frisch aus einem Block gebrochenes Material benutzt. Dabei muß jedoch in Rechnung gestellt werden, daß sich frisch gebrochenes Material bereits beim ersten Kontakt mit der Luft verändert (ZoBELL, 1972).

In der vorliegenden Arbeit sind allgemeine hydrographische und ökologische Ergebnisse enthalten. Befunde über spezielle Bewuchsunterschiede auf den untersuchten Natursteinsubstraten werden, nach pflanzlichen und tierischen Organismen getrennt, in zwei weiteren Veröffentlichungen erscheinen.

\section{METHODIK UND VERSUCHSDURCHFUHRUNG}

Das Wattenmeer erscheint durch seine geschürzte Lage für Bewuchsuntersuchungen vorteilhaft. Die diesem Lebensraum eigene Pflanzen- und Tierwelt ist relativ artenarm. Sie muß - bedingt durch den Gezeitenrhythmus - extremen Umwelteinflüssen angepaßt sein. Ein für Bewuchsuntersuchungen geeigneter Standort bot sich im Norden der Insel Sylt neben dem Lister Hafen am Anleger der dänischen Autofähre. Hier konnten,

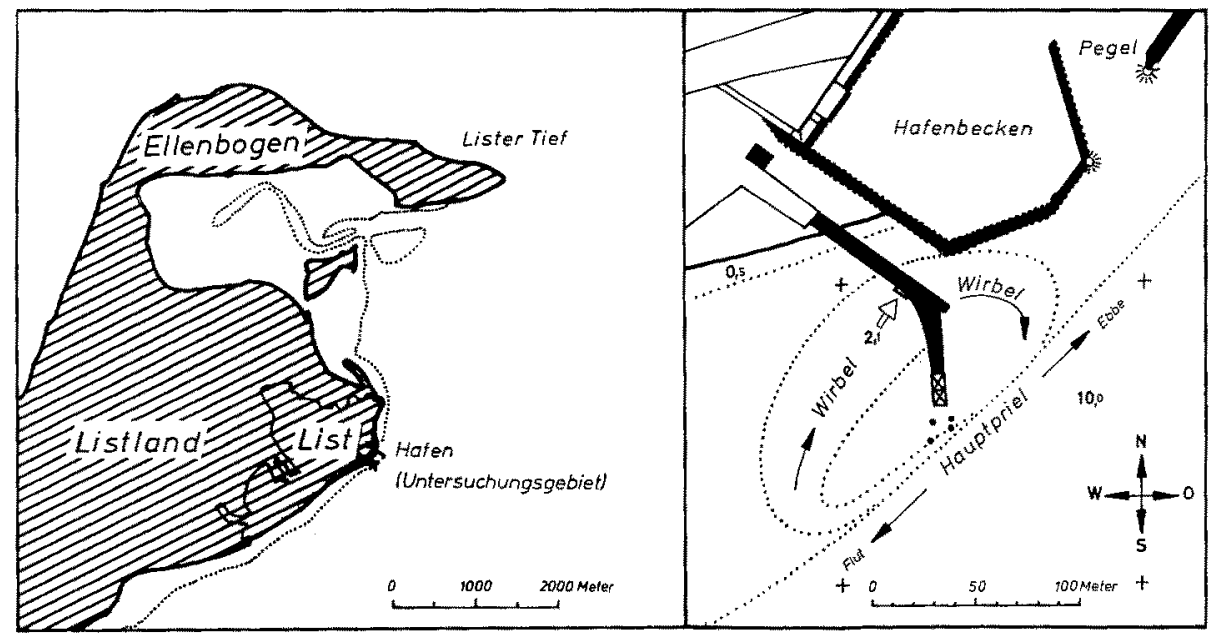

Abb. 1: Nördlicher Teil der Insel Sylt (Nordsee). Ausschnitt: Hafen von List mit dem Anleger der dänischen Autofähre. Pfeil $=$ Versuchsgestell 
unabhängig von einem Boot, Versuchsgestelle angebracht und davor Wasserproben für hydrographische Bestimmungen (Wassertemperatur, Salzgehalt, Planktonzusammensetzung) entnommen werden. Die Probenentnahme erfolgte zweimal täglich in der Mitte zwischen den Tiden, um Mirtelwerte des auf- und ablaufenden Wassers zu erhalten. Die Bewuchsuntersuchungen wurden 1971 und 1972 täglich von März bis Dezember durchgeführt (1973 zur Nachkontrolle während einer kürzeren Zeitspanne) und in der Litoralstation der Biologischen Anstalt Helgoland ausgewertet.

\section{Position des Versuchsgestells}

Das Versuchsgestell war am Bohlensteg der Anlegerbrücke derart angebracht, daß die Frontalseite nach Südwesten zeigte und von ca. 11 bis 17 Uhr direkte Sonnenbestrahlung erhielt. Bei den vorherrschenden westlichen Winden lag es im Windschatten des Listlandes (Abb. 1). Bei den selteneren östlichen Winden waren die Versuchssub-strate direkt der Brandung ausgesetzt. Der Tidenhub am Standort betrug 1,70 m, die Wassertiefe bei Niedrigwasser ca. 1,20 m. Der Hauptpriel, der die Masse des auf- und ablaufenden Wassers führt, zieht am Brückenkopf vorbei und bildet bei Flut einen Wirbel, der unter dem Bohlensteg hindurch wieder in den Hauptpriel mündet (Abb. 1, Ausschnitt). Das Versuchsgestell befand sich auf der Landseite des Wirbels, so daß es stets aus gleicher Richtung frontal angeströmt wurde.

\section{Substratanordnung}

Die Versuchssubstrate befanden sich als Reihen in 3 charakteristischen Bereichen zwischen den Tidenmarken: auf dem Hochwasser-Niveau (HW) mit der MitteltidenHodhwasserlinie als oberer Begrenzung (oberes Eulitoral), auf dem NiedrigwasserNiveau (NW) mit der Mitteltiden-Niedrigwasserlinie als untere Begrenzung (unteres Eulitoral) und auf dem Unterwasser-Niveau (UW), $75 \mathrm{~cm}$ unter der MitteltidenNiedrigwasserlinie (Sublitoral) (Abb. 2). Die Substrate waren in den genannten Bereichen derart nebeneinander angebracht, daß helle Natursteine (Solnhofener Plattenkalk, Muschelkalk), mitteltonige (Buntsandstein, Granit) und dunkle (Säulenbasalt, Basaltlava) aufeinanderfolgten. Die Substrate eines Farbbereiches unterschieden sich jeweils nach den Strukturmerkmalen fein-bis mittelkörnig und grobkörnig (Abb. 3).

Im ersten Versuchsjahr hatten sich auf Gesteinssubstraten mit naturrauher und den gleichen mit glatt gesägter Oberfläche in der Besiedlungsdynamik Differenzen ergeben, die sich auf die Verschiedenartigkeit der Oberfläche zurückführen ließen. Um die Wirkung der Oberflächenstruktur eingehend zu untersuchen, wurden künstlich hergestellte Substrate aus Quarzsand 5 abgestufter Korngrößen und Silizium-Karbid neben den Versuchsreihen aus Natursteinen eingesetzt (Abb. 3). Außer der Gegenüberstellung von Natursteinen und künstlichen Substraten erschien eine Einteilung in Langzeit- und Kurzzeitserien angebracht. An den Langzeitserien ließ sich die Sukzession der Besiedlung im Jahresablauf verfolgen. Die Kurzzeitserien verdeutlichten dagegen, 
wie hoch das "Angebot bodenreifer Keimlinge und Larven" war. Alle 8 bis 10 Tage wurde auf jedem Versuchssubstrat ein Feld von $122 \mathrm{~cm}^{2}$ mit Hilfe eines Kartiernetzes ausgezählt.

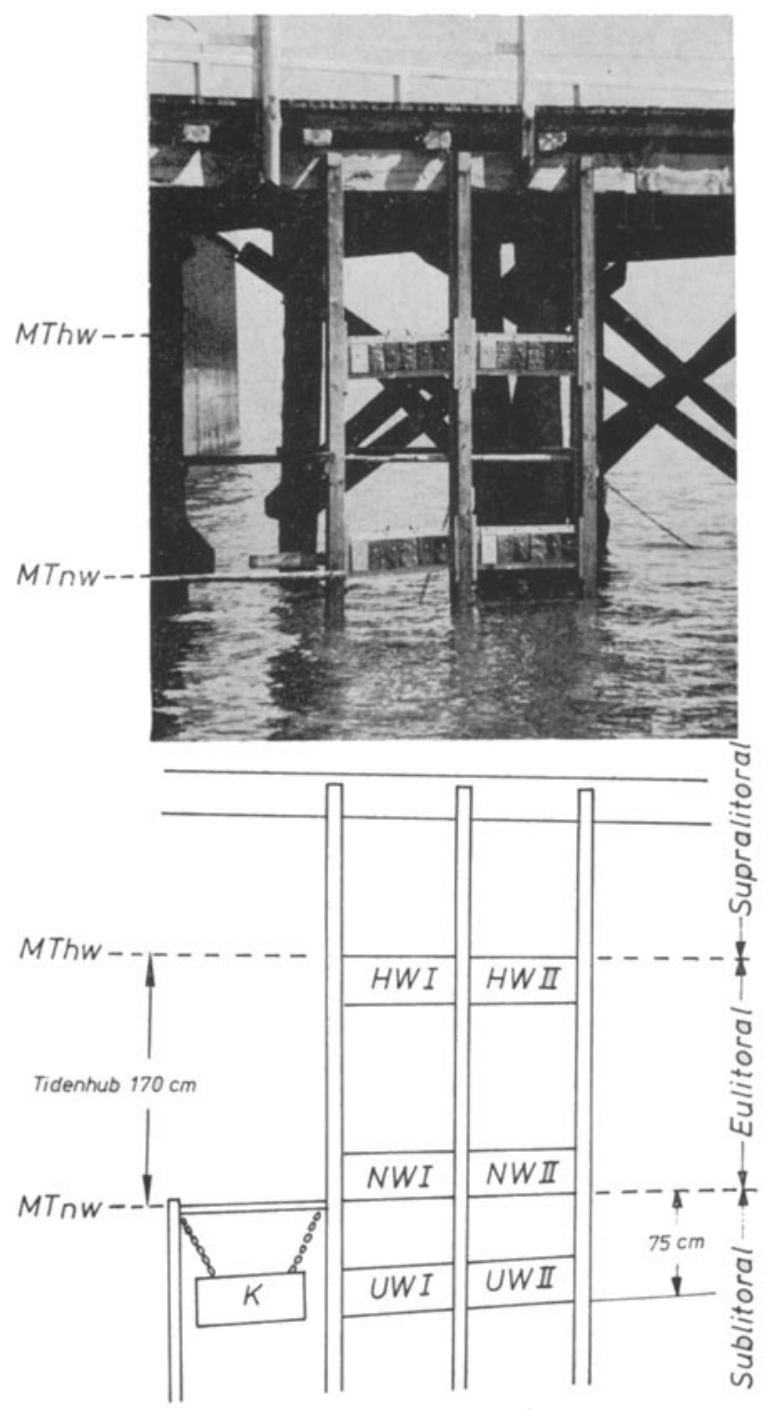

Abb. 2: Versuchsgestell unter In-situ-Bedingungen im Jahr 1971. Versuchsreihen auf dem Hochwasser-Niveau (HW), Versuchsreihen auf dem Niedrigwasser-Niveau (NW), Versuchsreihen auf dem Unterwasser-Niveau (UW), $\mathrm{I}=$ Langzeitserie, II $=$ Kurzzeitserie, $\mathrm{K}=$ Kontrollserie mit glattgesägten Gesteinssubstraten 


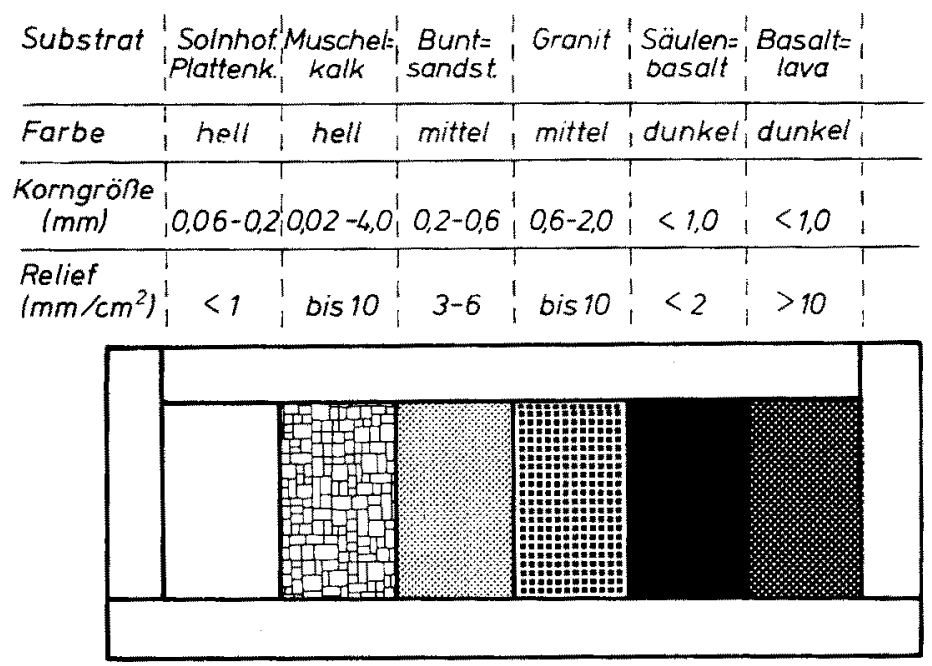

\begin{tabular}{|c|c|c|c|c|c|c|}
\hline \multirow[t]{2}{*}{ Substrat } & \multicolumn{5}{|c|}{ Quarzsand } & \multirow{2}{*}{$\begin{array}{l}\text { Silizium } \\
\text { Karbid }\end{array}$} \\
\hline & 1 & 1 & 1 & 1 & 1 & \\
\hline Farbe & i nell & hell & i hell & mittel & mittel & dunkel \\
\hline $\begin{array}{c}\text { Korngröle } \\
(\mathrm{mm})\end{array}$ & 0,25 & $0,25-0$ & $5,0,5-1,0$ & $1,0-2,0$ & $2,0-4,0$ & $2,0-4,0$ \\
\hline
\end{tabular}

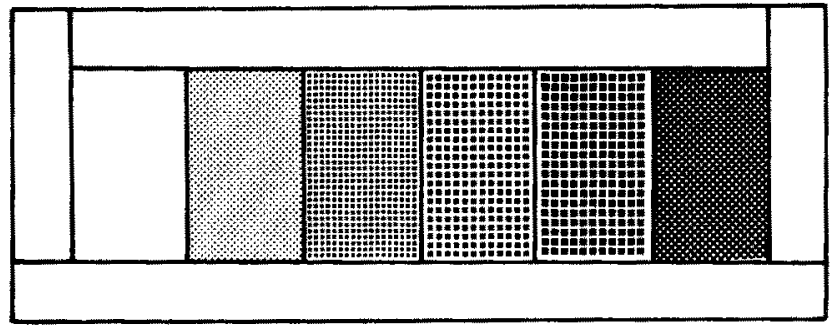

Abb. 3: Anordnung der Versuchssubstrate, Reihe mit Natursteinen (oben), Reihe mit Quarzsanden abgestufter Korngrößen (unten)

\section{ABIOTISCHE FAKTOREN}

\section{Wassertemperatur}

Der Gang der Wassertemperatur war in beiden Versuchsjahren verschieden. Anfang März 1971 erfolgte ein Kälteeinbruch, der das Watt zufrieren und die Wassertemperatur knapp unter $0^{\circ} \mathrm{C}$ sinken ließ. Noch im ersten A prildrittel betrug die Wassertemperatur (10-Tagesmittel) $2 \mathrm{C}^{\circ}$ weniger als in der entsprechenden Zeit des folgenden Jahres. Vermutlich wirkte sich dieser Kälteeinbruch verheerend auf einige 
Meeresorganismen aus. Planktonzusammensetzung und Besiedlung auf den Versuchssubstraten erschienen nach Art und Zeitpunkt des Auftretens wesentlich anders als 1972. Mitte Mai erwärmte sich das Wasser sprunghaft in wenigen Tagen und überschritt die Wassertemperatur (10-Tagesmittel) des folgenden Jahres in der entsprechenden Zeit um $2 \mathrm{C}^{\circ}$. Diese plötzliche Erwärmung nach einer langen kalten Periode schien die Massenentwicklung einiger meroplanktischer Formen auszulösen, der eine dichte Besiedlung auf den Substraten folgte. Das Maximum der Wassertemperatur erreichte Anfang Juli und Anfang August jeweils eine Höhe von $18,9^{\circ} \mathrm{C}$, während das Temperatur-Maximum im Juli 1972 fast $21^{\circ} \mathrm{C}$ betrug. Im Oktober 1971 dagegen war die Wassertemperatur von $13,5^{\circ} \mathrm{C}$ um $2 \mathrm{C}^{\circ}$ höher als im folgenden Jahr.

Nach den genannten Daten läßt sich 1971 als Jahr heftiger Temperaturschwan-

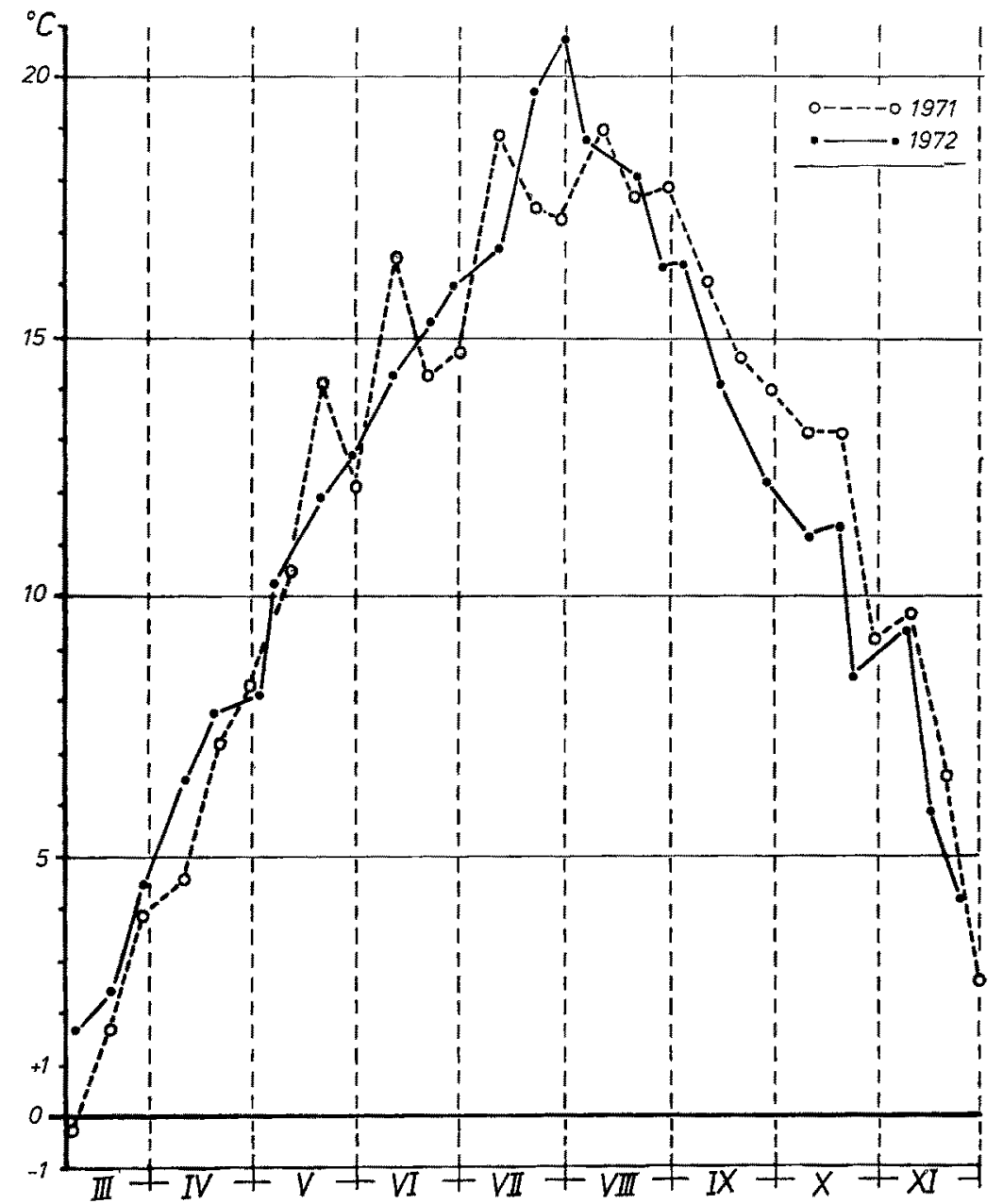

Abb. 4: Wassertemperaturen des auflaufenden Wassers in den Jahren 1971 und 1972 (10-Tagesmittel) 
kungen, bedingt durch einen offensichtlich größeren Wasseraustausch, charakterisieren. Das Jahr 1972 gestaltete sich im Durchschnitt wesentlich wärmer mit einem bis Ende Juli gleichmäßig ansteigenden und danach ebenso gleichmäßig absteigenden Temperaturverlauf (Abb. 4).

\section{Salzgehalt}

Der Salzgehalt der offenen Nordsee beträgt etwa $35 \%$. In den Küstengewässern schwankt er zwischen 30 und $33 \%$. Im allgemeinen war der Salzgehalt des auflaufenden Wassers geringfügig höher als der des ablaufenden Wassers, vor allem, wenn bei anhaltenden nordwestlichen. Winden mit der Flut höher konzentriertes Wasser aus der offenen Nordsee ins Wattenmeer eindringen konnte. Die Schwankungen im Jahres-

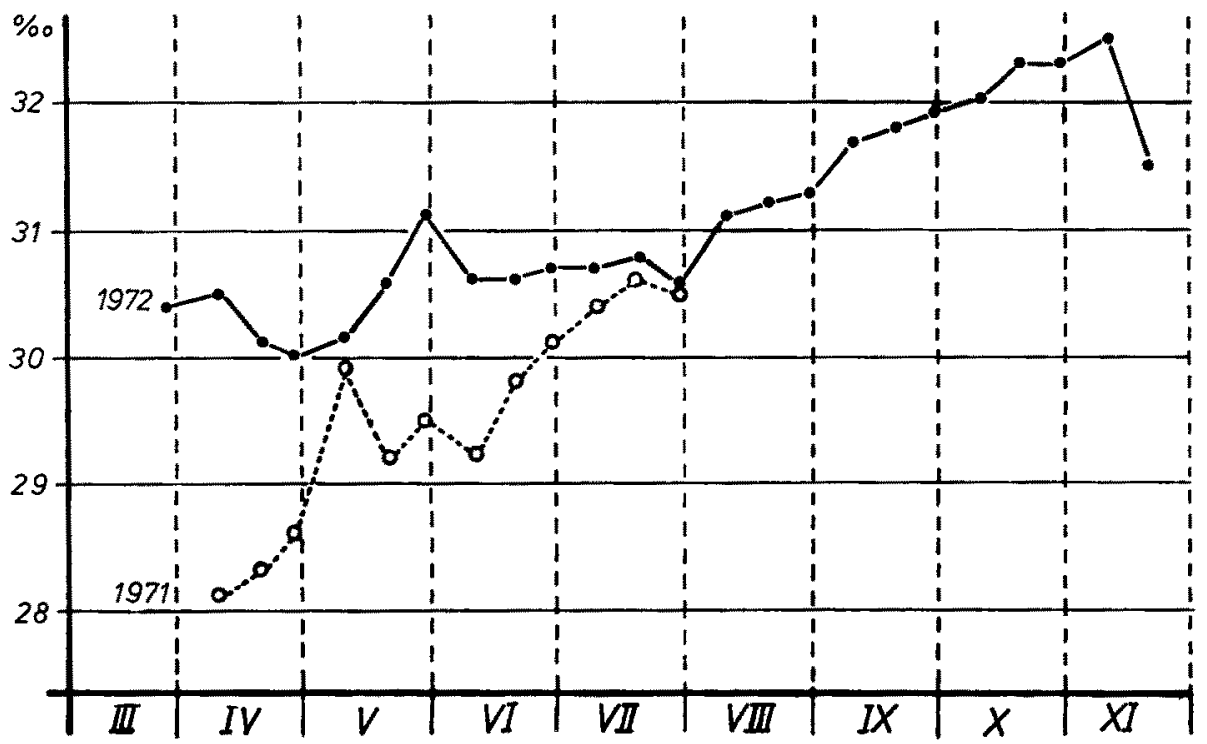

Abb. 5: Salzgehalt des auflaufenden Wassers in den Jahren 1971 und 1972 (10-Tagesmittel)

ablauf, die Flutstrom wie Ebbstrom gemeinsam waren, entsprachen den Darstellungen Goedeckes (1956) und Gessners (1957). In beiden Jahren lag das Minimum des Salzgehalts im April, bedingt durch die Schneeschmelze und der damit verbundenen höheren Süßwasserzufuhr der Flüsse. 1971 war der Salzgehalt nach dem Märzfrost um $2 \%$ niedriger als im folgenden Jahr. Er betrug durchschnittlich im April $28 \%$ und nahm bis zum Herbst ständig zu. Mitte Juli war der Salzgehalt mit 30,8\% in beiden Versuchsjahren gleich. Das Jahresmittel ergab einen Wert von ca. $31 \% \mathrm{~S}$ und stimmte mit der von GessNER (1957) angegebenen Isohaline überein (Abb. 5). 


\section{Trübungsgrad}

Der Trübungsgrad des Wassers beeinflußt vor allem lichtabhängige Algen. Die Sichttiefe des Wassers wurde im zweiten Versuchsjahr mit der Secchi-Scheibe ermittelt und daraus der Extinktionskoeffizient bestimmt. Der Monatsdurchschnitt der Sichttiefe betrug im Frühjahr und Herbst, bedingt durch Planktongehalt und aufgewirbelte Bodenpartikel, ungefähr $1 \mathrm{~m}$, im windstilleren Sommer dagegen mehr als $2 \mathrm{~m}$. Eine Ausnahme bildete der Juni, als sich mit dem Auftreten der Alge Phaeocystis ponchetii der Trübungsgrad des Wassers erhöhte (durchschnittliche Sichttiefe 1,30 m).

\section{BIOTISCHE FAKTOREN}

Die Art des Bewuchses auf den Versuchssubstraten hing von den im Untersuchungsgebiet siedelnden Organismen ab, deren Vermehrungsstadien durch das Wasser verbreitet wurden. Daneben traten Irrgäste auf, deren Larven der Tidenstrom aus einem für das Wattenmeer atypischen Biotop verfrachtet hatte, zum Beispiel die Cirripedier Balanus balanus und Verruca stroemia. Konkurrenten, Weidegänger und Räuber beeinflußten je nach Abundanz und Position die Entwicklung der Organismen auf den Versuchssubstraten.

\section{Die Lebensgemeinschaften des Untersuchungsgebietes}

Am Untersuchungsort sind zwei verschiedene Lebensgemeinschaften zu finden: eine ursprüngliche, für viele Bereiche des Wattenmeers typische Sandboden-Zönose, die Macoma-baltica-Gemeinschaft (Remane, 1940), und eine atypische HartbodenZönose, die entstand, als mit dem Bau des Hafens und des Fähranlegers künstlich ein Hartbodensubstrat geschaffen wurde. Der Sandboden am Untersuchungsort ist nicht bündig. Er wird ständig umgelagert, wie die starken Rippelmarken erkennen lassen. Der Besonderheit des Standorts angepaßt, hat sich eine Untergemeinschaft der Macomabaltica-Gemeinschaft entwickelt, die von Linke (1939) beschriebene Scoloplos-Variation mit den Leitformen Scoloplos armiger und Arenicola marina. Während der Versuchsjahre vermehrten sich räuberische Begleitformen wie Buccinum undatum, Carcinus maenas und Asterias rubens merklich, was nicht ohne Einfluß auf den Bewuchs der Versuchssubstrate blieb.

An den Brückenpfeilern selbst siedelten während der Versuchsjahre typische Vertreter einer Hartbodenflora und -fauna. Der Gezeitenhub, durchschnittlich 1,70 m, ist groß genug, um eine vertikale Zonierung erkennen zu lassen mit Supralitoral, der vorwiegend vom Klima beeinflußten Spritzzone, Eulitoral, dem Bereich zwischen Ebbeund Flutlinie, und Sublitoral, dem ständig untergetauchten Teil der Küste. Am Untersuchungsort dominierten Miesmuscheln und Balaniden über pflanzlichem Bewuchs. Der für das obere Eulitoral typische Fadenalgenrasen war spärlich entwickelt. Im Bereich der Mitteltiden-Niedrigwasserlinie traten breitblättrige Formen wie Enteromorpha linza, Ulva sp. und Porpbyra purpurea auf, wenige Dezimeter tiefer die buschigen 
Bildungen von Ceramium rubrum und Polysiphonia nigrescens. Fucus-Arten waren nicht vorhanden. Vereinzelte Exemplare siedelten sich lediglich 1975 nach 2 eisfreien Wintern zwischen Miesmuscheln an. $50 \mathrm{~cm}$ über dem Wattenmeerboden vernichtete Sandschliff jeglichen Pflanzenbewuchs.

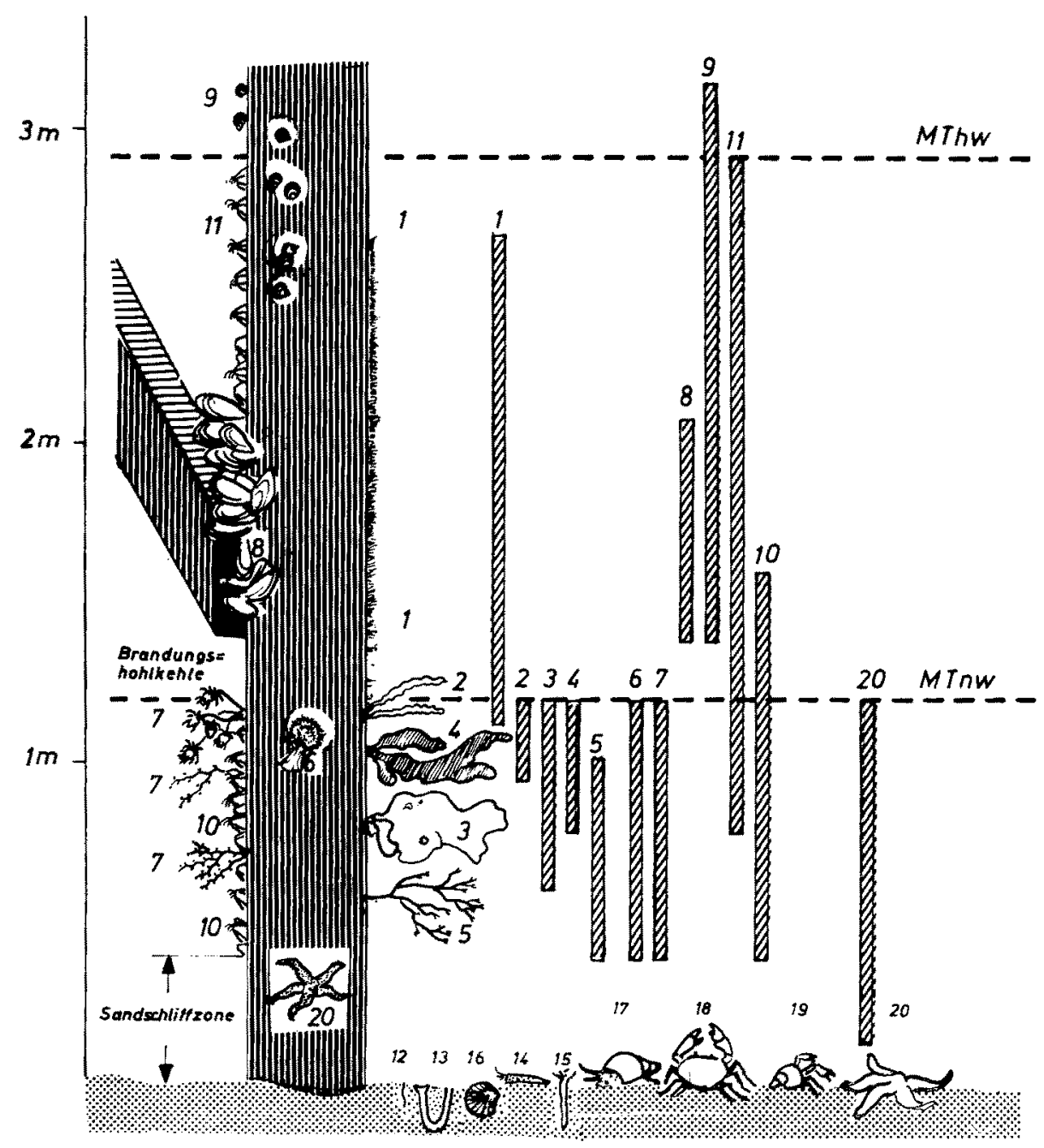

Abb. 6: Vertikale Zonierung und horizontale Verbreitung der Organismen in der unmittelbaren Umgebung des Versuchsgestells. Nr. 1 bis $11=$ Hartbodenflora und -fauna ( 1 Enteromorpha sp., 2 Enteromorpha linza, 3 Ulva lactuca, 4 Porphyra purpurea, 5 Ceramium rubrum, 6 Metridium senile, 7 Laomedea sp. und Tubularia larynx, 8 Mytilus edulis, 9 Littorina sp., 10 Balanus crenatus und $B$. improvisus, 11 B. balanoides); Nr. 12 bis $20=$ Scoloplos-Variation nach LINKE, 1939 (12 Scoloplos armiger, 13 Arenicola marina, 14 Pectinaria corveni, 15 Lanice conchilega, 16 Cardium edule, 17 Buccinum undatum, 18 Carcinus maenas, 19 Eupargurus bernbardus, 20 Asterias rubens). Die generelle Zonierung der Organismen ist durch schraffierte Vertikalkolumnen angedeutet 
Die vertikale Verbreitung der tierischen Organismen war besonders deutlich abzugrenzen (Abb. 6). Die Zone der Balaniden erstreckte sich als gleichmäßiger Überzug von der Mitteltiden-Hochwasserlinie bis zum bewuchsfreien Streifen über dem Wattenmeerboden und bot nur in der Brandungshohlkehle dicht über der Mitreltiden-Niedrigwasserlinie ein wechselhaftes Bild zerstörter alter und junger Siedlungen. Unterhalb der Brandungshohlkehle herrschten Balanus crenatus und B. improvisus vor. Dazwischen entfalteten sich Seeanemonen und Hydrozoenkolonien, bewegten sich Seeigel und Seesterne als Weidegänger und Räuber. Oberhalb der Brandungshohlkehle dominierte B. balanoides. Zu ihm gesellte sich im extrem warmen Sommer 1975 (mittlere Wassertemperatur im August $20^{\circ} \mathrm{C}$ ) der aus Australien eingeschleppte Balanide Elminius modestus. Die Balaniden wurden an geschützten Stellen vollkommen von Mytilus edulis verdrängt, die in den Winkeln zwischen Brückenpfeilern und Querverstrebungen kleine dichte Muschelsiedlungen bildete. Auf die Zone der Balaniden folgte ein Littorinen-Streifen. Dieser überlappte die obere Verbreitungsgrenze der Balaniden und schloß den Zonenaufbau ab. Mit einer Breite, die je nach Jahreszeit und Tidenhöhe wechselte, reichte der Littorinen-Streifen über die Mitteltiden-Hochwasserlinie hinaus.

\section{Wechselbeziehungen $\mathrm{zw}$ ischen Plankton- und Bewuchsorganismen}

Fast alle Bewuchsorganismen besitzen Entwicklungsstadien, die über eine kürzere oder längere Zeitspanne hinweg als sogenannte meroplanktische Formen im Meerwasser enthalten sind, bevor sie zum Bodenleben übergehen. Die Zusammensetzung des Planktons zeigt jedoch nicht nur die zu erwartende Art, Dichte und den Zeitpunkt der Besiedlung auf den Substraten an, sondern ist auch als Nahrungsgeber für die weitere Entwicklung fischender und strudelnder Bewuchsorganismen maßgeblich. Ebenso läßt sich aus der Zusammensetzung des Planktons der Einfluß konkurrierender, weidender und räuberischer Formen ungefähr bestimmen.

Die Wechselbeziehung zwischen Bewuchs und Zusammensetzung des Planktons machten am Untersuchungsort eine ständige Kontrolle des Meerwassers auf seinen Gehalt an Organismen notwendig. Zweimal täglich ( $3 \mathrm{~h}$ nach Niedrigwasser und $3 \mathrm{~h}$ nach Flut) wurden je 101 Meerwasser an der Oberfläche geschöptt, durch ein Netz von $150 \mu$ Maschenweite gefiltert und der Siebrückstand unter einem Binokular ausgezählt. Da nanoplanktische Formen, zum Beispiel Vermehrungsstadien der Algen, beim Filtern die Netzmaschen passierten, ließen sich nur größere Exemplare des Phyto- und Zooplanktons erfassen. Eine Quadrateinteilung erleichterte das Auszählen. Die Artbestimmungen erfolgten nach JoHNSTONE et al. (1924) und THORSON (1946).

\section{Einfluß von Konkurrenten, Weidegängern und Räubern}

Die Struktur vieler Lebensgemeinschaften beruht auf der Wechselbeziehung von Konkurrenten (CONNEL, 1972) und wird zum Beispiel von räuberischen Formen wie dem Seestern geprägt (Panne, 1974). Connell (1961), Medows (1969), Newell (1970) 
und FOSTER (1971) berichten über Raumkonkurrenzen innerhalb einer Balanidensiedlung oder $z$ wischen den Individuen verschiedener Arten. Solche Beispiele von Raumkonkurrenzen waren fortlaufend auf den Versuchssubstraten zu verfolgen. Überzog ein dichter, feinfädiger Grünalgenrasen die Gesteinsoberfläche, fanden die bodenreifen Cypris-Larven der Balaniden keine Anheftungsstellen und suchten zum Siedeln schattige Mulden und Überhänge auf, wo die Grünalgen aus Lichtmangel schlecht gedeihen konnten. Zwischen der großblättrigen Rotalge Porphyra purpurea dagegen, die sich langsamer und weniger dicht entwickelte, blieb genügend Raum für ein Anheften der Cypris-Larven erhalten.

In den Balanidensiedlungen ließen sich auch eindrucksvolle Beispiele intraspezifischer Konkurrenz beobachten. So wurden Jungtiere, die sich zwischen ca. 3 Wochen älteren Individuen derselben Art angesetzt hatten, durch den Wachstumsdruck derer Basalplatten zusammengeschoben, deformiert und schließlich abgehoben (vgl. GuTMANN, 1960). Der gleiche Vorgang spielte sich bei Individuen zweier verschiedener Balanidenarten ab. Während der Versuchsjahre war die hohe Sterblichkeit der Jungtiere unter anderem auf diese Form der Raumkonkurrenz zurückzuführen.

Pflanzenfressende Weidegänger wie Littorina littorea traten ïberraschenderweise selten auf den Versuchssubstraten auf. Vermutlich bereitete die spezielle Anordnung der Versuchsreihen den überwiegend vertikal wandernden Schnecken Schwierigkeiten,

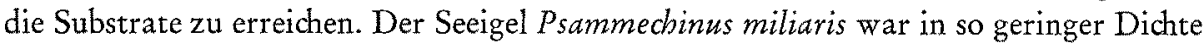
vorhanden, daß er den Pflanzenbewuchs nicht wirksam reduzieren konnte.

Als Weidegänger auf Hydrozoenkolonien waren die Nacktschnecken Tergipes despectus, Dendronotus frondosus, Cratena aurantia und Facellina drummondi, der Pantopode Phoxichilidium femoratum anzutreffen. Sie verminderten die Polypenzahl und brachten die Kolonien zum Kümmern. Die Freßtätigkeit von $T$. despectus ließ sich an den Rückenpapillen ablesen. Diese färbten sich je nach Art der Nahrungsaufnahme einmal bräunlich (zur Zeit des Entwicklungsmaximums von Laomedea sp.) oder orangerot, wenn Tubularia larynx auf den Substraten dominierte (Hoffmann, 1936).

Die Jungtiere des Seesterns Asterias rubens ernähren sich von zum Bodenleben übergehenden Miesmuscheln und Balaniden (HaNcock, 1955). Sie vernichteten auf den Versuchssubstraten unterhalb der Mitteltiden-Niedrigwasserlinie die Frühjahrsbesiedlung der Balaniden bis auf wenige Exemplare, die sich im Schutz tiefer Spalten oder Tubularia-Kolonien angesetzt hatten. Die räuberische Tätigkeit des Seesterns bestimmte, wie bereits PAINE (1974) nach mehrjährigen Experimenten nachwies, auch auf den Versuchssubstraten die untere Grenze der Miesmuscheln- und Balanidenverbreitung. Sie reichte bis wenig unter die Mitteltiden-Niedrigwasserlinie. Die Zerstörung durch den Seestern schien im Sublitoral tiefgreifender zu sein als die durch die Wirkung abiotischer Faktoren.

\section{UNTERSUCHUNGSERGEBNISSE UND SCHLUSSFOLGERUNGEN}

Während beider Versuchsjahre zeigten sich wesentliche Unterschiede im Zeitpunkt des Auftretens und in der Dichte der Planktonorganismen. Die entsprechenden Unterschiede wurden ebenfalls sichtbar, wenn die bodenreif gewordenen Stadien meroplank- 
tischer Formen auf den Versuchssubstraten zu siedeln begannen. Um den Zusammenhang zwischen Planktonorganismen und Bewuchs zu verfolgen, werden die Befunde beider Bereiche - im Vergleich zu den Temperaturschwankungen der Versuchsjahre gegenübergestellt.

\section{Veränderungen des Planktons in Abhängigkeit von Jahreszeit und mittlerer Wassertemperatur}

Perio de März/A pril (Abb. 7). Der Märzfrost 1971, verbunden mit dem niedrigen Salzgehalt des Meerwassers, bedeutete für die Entwicklung einiger planktischer Organismen eine „kleine Katastrophe". Die Schwarmbildung holoplanktischer Copepoden (Acartia clausi, Temora longicornis, Centropagus bamatus und Pseudo-

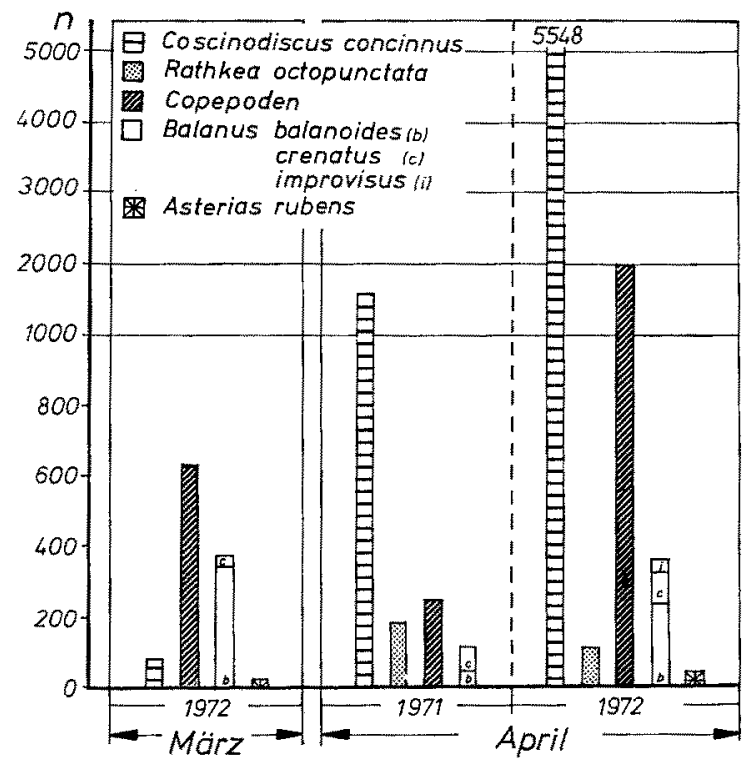

Abb. 7: Abundanz einiger häufiger Plankton-Organismen im März/April 1971 und 1972 (Monatssummen, entnommen aus täglich 2 Meerwasserproben von je 10 1)

calanus elongatus) und die Menge der frühen Balanidenbrut, vor allem von Balanus balanoides, war äußerst gering. Das Plankton erwies sich bis Mai individuenärmer als 1972. Die Anzahl der genannten Copepoden erreichte nach einem milden Winter im April 1972 das Zehnfache des Vorjahres, die der Balanidenlarven das Fünffache. Der Anteil von $B$. crenatus war in beiden Jahren ungefähr gleich. Vermutlich hatte der Eisschliff viele Elterntiere von $B$. balanoides vernichtet, bevor jene ihre Brut entlassen konnten. Der in größerer Tiefe lebende $B$. crenatus wurde dagegen weniger davon betroffen. Die Hydromeduse Ratbkea octopunctata, die dem Winterplankton angehört, trat im April 1971 wesentlich häufiger auf als nach dem milden Winter 1972. 
Periode Mai/Juni (Abb. 8). Auf den plötzlichen Temperaturanstieg im Mai 1971 folgte eine explosionsartige Entwicklung einiger Plankter. Bei Vertretern des Phytoplanktons, der Diatomee Coscinodiscus concinnus und der Alge Phaeocystis pouchetii, trat eine Massenvermehrung ein, die bis Ende Juni anhielt. Entwicklungshöhe und -dauer wurden von beiden Arten 1972 nicht mehr erreicht. Während sich beim Zooplankton die Anzahl der Copepoden und Nauplien borealer Balaniden

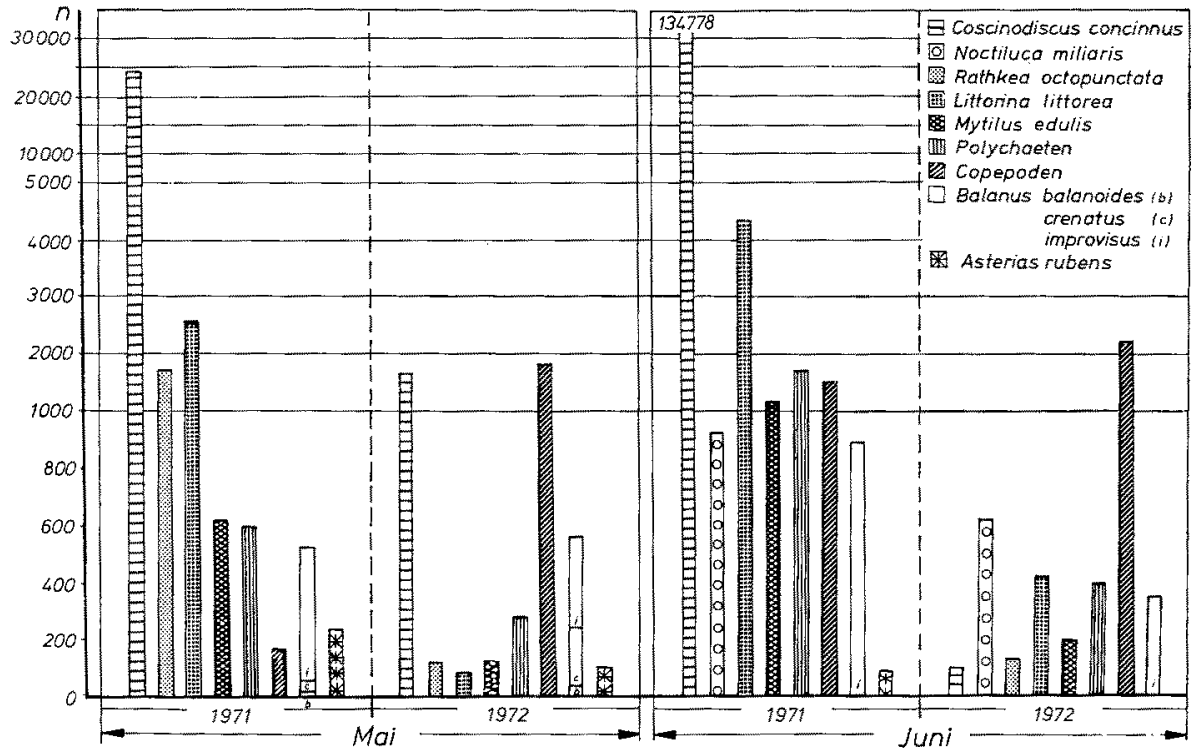

Abb. 8: Abundanz einiger häufiger Plankton-Organismen im Mai/Juni 1971 und 1972 (Monatssummen, entnommen aus täglich 2 Meerwasserproben von je 10 1)

(Balanus balanoides und B. crenatus) weiter verringerte, setzte eine starke Vermehrung von Ratbkea octopunctata, der Strandschnecke Littorina littorea, der Miesmuschel Mytilus edulis, einiger Spioniden und von Balanus improvisus ein. Diese Entwicklung dauerte den Juni über an. Einzig Rathkea octopunctata war in dem Monat nicht mehr vorhanden, nachdem das Wasser die für die Medusenfortpflanzung begrenzende Temperatur von $15^{\circ} \mathrm{C}$ erreicht hatte (WERNER, 1958). 1972 dagegen traten die genannten Arten zahlenmäßig im Plankton zurück. An ihrer Stelle hatten Copepoden das Übergewicht.

Nachdem vermutlich klimatische und hydrographische Einflüsse in Mai 1971 die Vermehrung der genannten Organismen begünstigten, könnte die ungestörte Weiterentwicklung der Larven unter anderem darauf zurückzuführen sein, daß - vielleicht bedingt durch den vorausgegangenen Märzfrost - Nahrungskonkurrenten, filternde und räuberische Plankton- und Benthosformen reduziert waren. Dementsprechend müßte 1972 nach dem eisfreien Frühling ein vollkommen anderes Verhältnis zwischen Larven und ihren Konkurrenten bestanden haben, was zu einer höheren Verlustrate der ersteren führte. Als Konkurrenten könnten 1972 die zahlreichen Copepoden und 
benthischen Filtrierer wie Miesmuscheln und Balaniden angesehen werden. Sie mögen dazu beigetragen haben, mehr als 1971 die planktischen Larvenstadien zu dezimieren.

Periode Juli/August (Abb. 9). Während der warmen Monate Juli/ August bestimmten in beiden Jahren der Dinoflagellat Noctiluca miliaris und Copepoden den Gehalt des Zooplanktons. Entsprechend einer mittleren Wassertemperatur, die 1972 um $1 \mathrm{C}^{\circ}$ höher lag als im Vorjahr, trat Noctiluca miliaris besonders häufig auf.

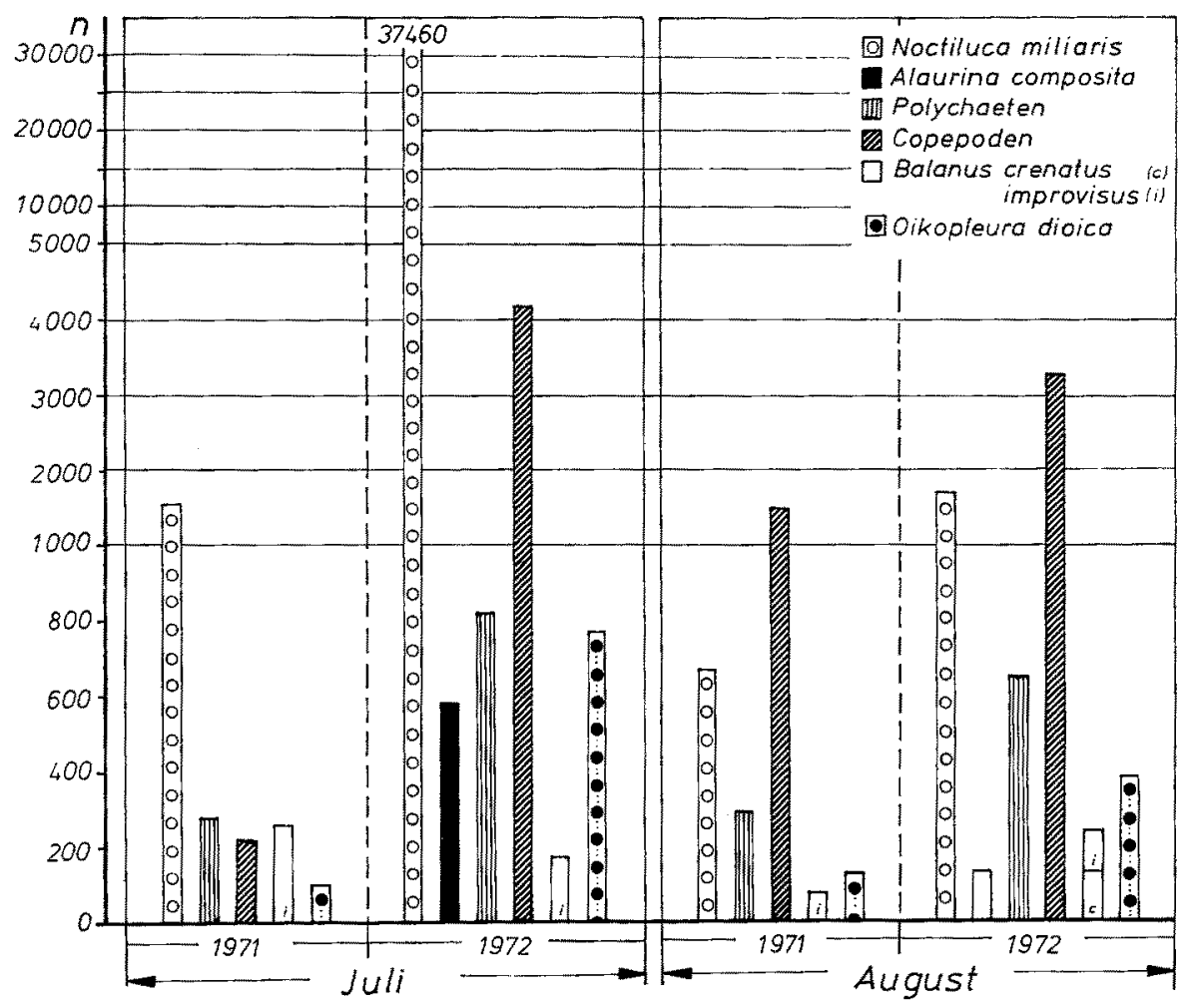

Abb. 9: Abundanz einiger häufiger Plankton-Organismen im Juli/August 1971 und 1972 (Monatssummen, entnommen aus täglich 2 Meerwasserproben von je 101)

Auch der wärmerem Wasser angepaßte Tunicat Oikopleura dioica war zahlreicher vorhanden mit einem Entwicklungsmaximum bei ca $20^{\circ} \mathrm{C}$.

Unter den Organismen, die regelmäßig in den. Wasserproben vorkamen, nahm Alatrina composita eine Sonderstellung ein. Dieser einzige echte Plankter unter den Turbellarien des Nordseebereichs vermehrt sich intensiv durch Querteilung, wenn seine Hauptnahrung in warmen Sommern ausreichend vorhanden ist (Hofker, 1930). Mit der sogenannten Jütlanddrift soll er an die dänische Küste gelangen. 1971 ließ sich A. composita nicht mit Bestimmtheit nachweisen; 1972 dagegen war sie Ende Juli, als sich das Wasser über $20^{\circ} \mathrm{C}$ erwärmte, besonders häufig. Dieses kurzfristige Vorkommen des Turbellars - es trat im auflaufenden Wasser auf - schien darauf hinzudeuten, 
daß bei zugleich yorherrschenden westlichen Winden ein anderer Wasserkörper ins Wattenmeer eindrang. Vor und nach dieser Juliwoche war $A$. composita bei überwiegend östlichen und nördlichen Winden jeweils vereinzelt zu finden.

Zusammen mit dem Wasserkörper, der Alaurina composita verfrachtete, gelangten Larven von Polydora ciliata, vermutlich die Brut einer ersten Generation, ins Wattenmeer und bestimmten den Besiedlungsbeginn dieses Polychaeten auf den Versuchssubstraten (August 1972). Dagegen hatte die Besiedlung $1971 \mathrm{ca} .2$ Monate früher eingesetzt, da die Larven entsprechend früher (Mai 1971) im Plankton enthalten waren. Nach den um diese Zeit vorherrschenden nördlichen und östlichen Winden zu urteilen, könnten die Larven, vermutlich die Brut einer iberwinterten Generation, mit einem anderen Wasserkörper als 1972 in den Bereich des Untersuchungsorts gelangt sein.

Die sogenannte "Herbstblitte" des Planktons machte sich im Untersuchungsgebiet in beiden Jahren nur unwesentlich bemerkbar.

Einfluß der Wassertemperatur auf die Abundanz zooplanktischer Formen in den Jahren 1971 und 1972

Nach den Untersuchungen auf den Gehalt an zooplanktischen Organismen ließ sich 1971 als Jahr mit überwiegend meroplanktischen Formen, 1972 als Jahr mit überwiegend holoplanktischen Arten charakterisieren (vgl. HickeL, 1975). In Tabelle 1 ist der Anteil der Plankter beider Jahre gegenübergestellt.

Tabelle 1

Anzahl tierischer Holo- und Meroplankter in den Jahren 1971 und 1972. Gesamtindividuenzahl, entnommen aus zweimal täglich gewonnenen 10-1-Meerwasserproben (März bis Dezember)

\begin{tabular}{|lrr|}
\hline \multicolumn{1}{|c}{ Organismen } & 1971 & 1972 \\
\hline Noctiluca miliaris & 3883 & 53405 \\
Copepoden & 6201 & 12675 \\
Cladoceren & 116 & 394 \\
Tunicaten & 369 & 1799 \\
\hline Summe der Holoplankter & 10619 & 75273 \\
\hline Ratbkea octopunctata & 1559 & 199 \\
Postlarven von Littorina littorea & 7319 & 668 \\
Postlarven von Mytilus edulis & 1879 & 758 \\
Polychaetenlarven & 3399 & 2863 \\
Balanidenlarven & 2222 & 1997 \\
\hline Summe der Meroplankter & 16378 & 6485 \\
\hline
\end{tabular}

Als Ursache für die Populationsunterschiede beider Jahre ließen sich klimatische und hydrographische Einflüsse vermuten. Der Kälteeinbruch im März 1971 und der darauf folgende sprunghafte Anstieg der Wassertemperatur im Mai mochten das Verhältnis zwischen Organismen, ihrer Nahrung, ihren Konkurrenten und Feinden derart verschoben haben, daß sich die Populationen vollkommen anders entwickelten als im 
folgenden Jahr mit seinen ausgeglichenen klimatischen und hydrographischen Bedingungen. Als charakteristisches Beispiel für temperaturbedingte Populationsverschiebungen kann das Nauplien-Verhältnis der Balanidenlarven herangezogen werden. Die Gesamtzahl der Balanidenlarven erwies sich in beiden Versuchsjahren als annähernd gleich. Wurden jedoch die Anteile von Balanus balanoides und $B$. crenatus denen von $B$. improvisus gegenübergestellt, ergab sich folgende Relation (Tabelle 2):

Tabelle 2

Nauplien von Balantts balanoides, B. crenatus und B. improvisus in den Jahren 1971 und 1972. Anzahl der Individuen, entnommen aus zweimal täglich gewonnenen 10-1-Meerwasserproben (März bis Dezember)

\begin{tabular}{|lcc|}
\hline \multicolumn{1}{|c|}{ Nauplien } & 1971 & 1972 \\
\hline $\begin{array}{l}\text { Balanus balanoides } \\
\text { einzige Brut im Frühjahr }\end{array}$ & 70 & 625 \\
$\begin{array}{l}\text { Balanus crenatus } \\
\text { Fruhjahrsbrut }\end{array}$ & 120 & 324 \\
Herbstbrut & 386 & 158 \\
Balanus improvisus & 1646 & 890 \\
\hline
\end{tabular}

Balanus balanoides und $B$. crenatus, deren Nauplien bereits im frühen Frühjahr auftreten, waren nach der Märzkälte 1971 weitaus seltener als im frostfreien März 1972 im Plankton enthalten (zum Beispiel durch die Vernichtung der Elternstöcke durch Eisschliff, bevor sich die Larven fertig entwickelt hatten). Auf $B$. improvisus, dessen Nauplien später als die vorgenannten Arten ins Meerwasser entlassen werden, wirkte sich der plötzliche Temperaturanstieg im Mai vermutlich günstig aus. SouTHWARD (1957) bezeichnet diese Spezies als eine an kaltes Klima angepaßte Warmwasserart. Im September 1971, dessen mittlere Wassertemperatur um $2 \mathrm{C}^{\circ}$ höher lag als 1972, bildete $B$. crenatus eine zahlreiche zweite Brut aus. Es befanden sich doppelt so viel Nauplien im Plankton als im Herbst des folgenden Jahres. Neben den positiven hydrographischen Einflüssen schien die Wirkung eines biotischen Faktors die Zabl der Balanidenlarven zu bestimmen. Die Jungtiere von Asterias rubens, die sich unterhalb der Mitteltiden-Niedrigwasserlinie von Balaniden (hauptsächlich B. crenatus und B. improvisus) ernähren, hatten 1 Monat später als 1972 die Metamorphose beendet, so daß sie die Elternstöcke erst dezimieren konnten, nachdem die Brut reif geworden war.

Wie bei der Ausbildung der Herbstbrut von Balanus crenatus, wo sich im Zusammenhang mit der Wassertemperatur das Räuber-Beuteverhältnis verschob, mögen ähnliche biotische Faktoren die Entwicklung planktischer Formen beeinflußt haben. So war mit der Individuenarmut des Planktons nach dem Märzfrost 1971 auch die Zahl der Nahrungskonkurrenten, fischenden und räuberischen Organismen herabgesetzt. Als sich im Mai das Wasser plötzlich erwärmte und damit eine intensive Vermehrung der Larven von Littorina littorea, Mytilus edulis, Polychaeten und Balanus improvisus einsetzte, blieb die Verlustrate unter den larvalen Entwicklungsstadien entsprechend niedrig. Im individuenreichen Plankton des Jahres 1972 dagegen - nach einem milden 
Winter enthielt es in der Mehrzahl Copepoden, und die fischenden Benthosformen waren nicht durch Eisgang geschädigt - verringerten sich die Oberlebenschancen der Larven. Das Übergewicht larvaler Stadien im Zooplankton 1971 könnte demnach durch Eisgang, niedrige Wassertemperaturen im März und ein dadurch bedingtes Fehlen von Nahrungskonkurrenten, fischenden und räuberischen Organismen gedeutet werden.

\section{Populationsunterschiede der Bewuchsorganismen in den Jahren 1971 und 1972}

Organismen, die auf den Versuchssubstraten siedelten, reichten von Bakterien über Pflanzen bis zu wirbellosen Tieren. Als Primärbesiedler traten Mikroorganismen (Bakterien, Pilze, einzellige Algen, Protozoen) auf. Diese Formen bilden im allgemeinen einen Besiedlungsrasen, den sogenannten „Primärschleim“. Der Primärschleim wurde bei den Untersuchungen nicht berücksichtigt. Unter den makroskopischen Sekundärbesiedlern war $z w i s c h e n$ häufigen und seltenen Arten zu unterscheiden. Die häufigen Arten umfaßten folgende Formen: Ulva lactuca, Enteromorpha sp., Ulothrix sp., Urospora sp. (Grünalgen); Bangia fuscopurpurea, Porphyra purpurea, Ceramium rubrum (Rotalgen); Tubularia larynx, Coryne sp., Laomedea flexuosa, L. longissima (Hydrozoa); Mytilus edulis (Bivalvia); Polydora ciliata, Lanice conchilega (Polychaeta); Balanus balanoides, $B$. crenatus, B. improvisus (Crustacea). Sie waren ausnahmslos typisch für den untersuchten Lebensraum.

Abbildung 10 zeigt Differenzen in Besiedlungsbeginn und -dichte. Es ist nicht zu übersehen, daß die Besiedlung 1971 allgemein später einsetzte als 1972, offensichtlich bedingt durch Unterschiede in der mittleren Wassertemperatur während der Wintermonate beider Jahre. Formen des frühen Frühjahrs, zum Beispiel Balanus balanoides, traten, wie auch seine Nauplien im Plankton, nach dem Märzfrost 1971 spärlich auf. Formen des späteren Frühjahrs, zum Beispiel Enteromorpha sp., Laomedea longissima, Mytilus edulis, Polydora ciliata und $B$. improvisus, erlebten nach einem raschen Temperaturanstieg desselben Jahres ein Maximum der Besiedlung, das 1972 nicht mehr erreicht wurde. Dagegen entwickelte sich 1972 der Algenbewuchs üppiger. B. balanoides setzte sich nach einem milden März (Monatsmittel der Wassertemperatur $3,6^{\circ} \mathrm{C}$ ) zeitiger und zahlreicher als im Vorjahr an, und die Besiedlung der oben genannten Tierformen erfolgte früher, jedoch weniger intensiv. Eine große Populationsdichte erreichten in den besonders warmen Sommermonaten desselben Jahres dagegen Arten, die sich bei höheren Wassertemperaturen vermehren können, wie Tubularia larynx (Pyefinch \& Downing, 1949), Laomedea flexuosa (Crowell, 1953) und Lanice conchilega (Kessler, 1963). Die Besiedlungsbefunde stimmten mit den Ergebnissen der Planktonauszählung überein.

Außer den angeführten häufigen Arten sind noch folgende sessile Vertreter der Makrofauna gefunden worden: die Seeanemonen Sagartia sp. und Metridium senile (im August häufig); vereinzelt die Hydrozoe Podocoryne carnea (November); gelegentlich Kolonien des Moostierchens Electra pilosa und Kamptozoen (September). An hemisessilen und vagilen Formen waren vertreten: Nematoden, Polychaeten (darunter Phyllodoce sp. und Lepidochiton squamatus), Harpacticiden und Amphipoden 
in großer Zahl, vereinzelt der Flohkrebs Jassa pulchella, die Käferschnecke Lepidochiton cinereus und die Pantoffelschnecke Crepidula fornicata. Zusammen mit Tubularia larynx erschien im September Caprella linearis in nicht abzuschätzender Menge. Diese Organismen waren meist Sekundärbesiedler 2. Ordnung. Sie folgten zeitlich auf die Sekundärbesiedler 1. Ordnung, den "Pionieren" (DEN HARTOG, 1959), wie flächenbedeckenden Grün- und Rotalgen, Hydrozoenkolonien und Balanidenpopulationen. Da

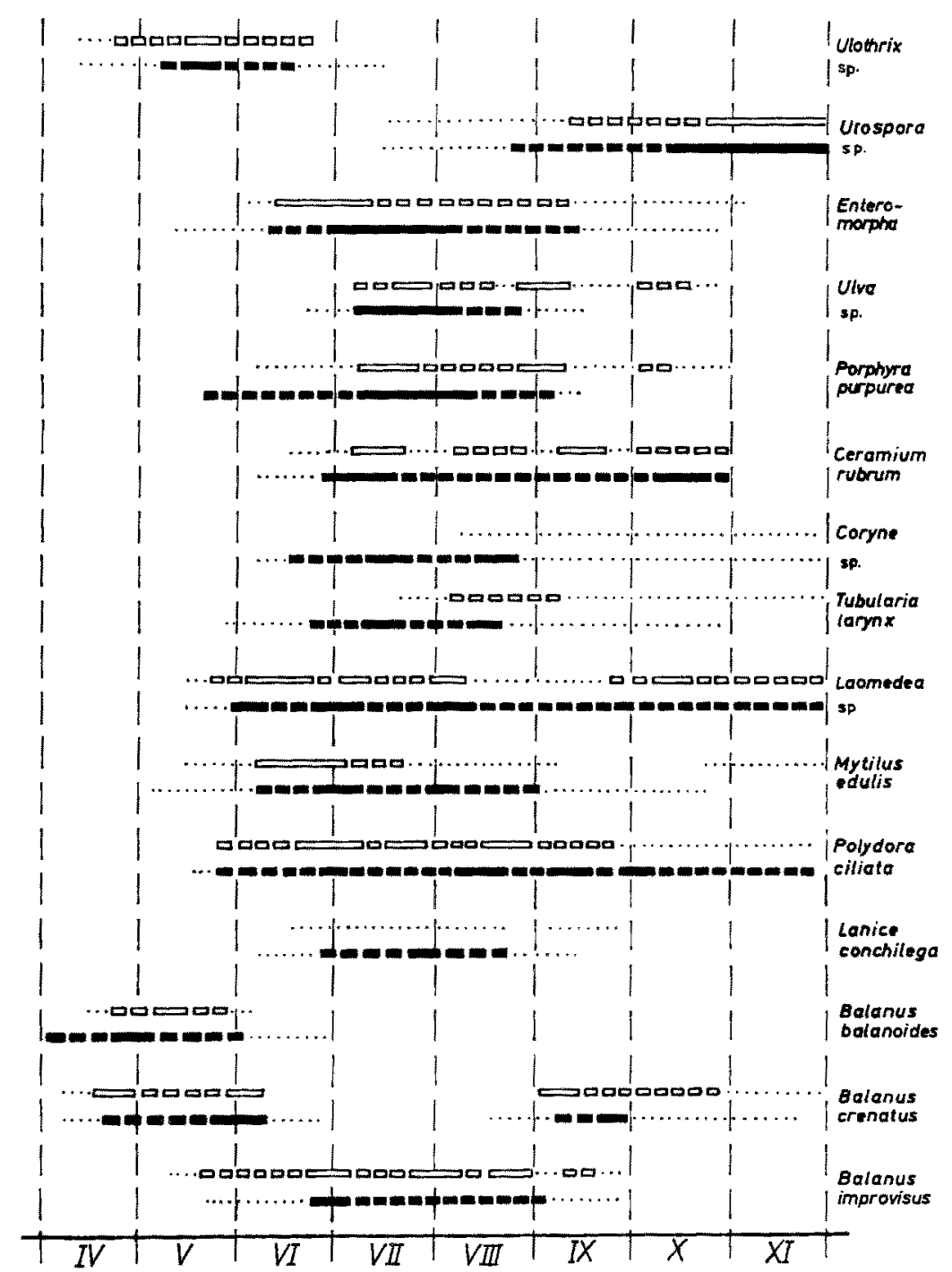

Abb. 10: Besiedlungsdynamik häufiger Bewuchsorganismen $1971 \square$ und 1972 punktierte Linie: $<20$ Individuen $122 \mathrm{~cm}^{-2}$; gestrichelte Linie: $>20$ Individuen $\mathrm{cm}^{-2}$; durchgezogene Linie: maximale Populationsdichte 
der winterliche Eisgang den Bewuchs in jedem Jahr wieder zerstörte, mußte stets eine neue Besiedlung durch Pioniere erfolgen.

Neben den aufgezählten Formen sind die Cirripedier Verruca stroemia und Balanus balanus besonders zu erwähnen. Sie wurden nach Aussage von Herrn Dr. ZIEGeLMEIRR (Biologische Anstalt Helgoland, Litoralstation in List) im Untersuchungsgebiet erstmalig nachgewiesen und als Irrgäste eingestuft. Beide Arten siedelten dicht unter der Mitteltiden-Niedrigwasserlinie, V. stroemia im Oktober, B. balanus im Frühjahr. Letzterer erreichte bis November einen basalen Durchmesser von $17 \mathrm{~mm}$. Der Balanide Elminius modestus, der sich in den letzten 10 Jahren entlang der Nordseeküste bis Dänemark ausgebreitet hat (KÜHL, 1954; DEN HARTOG, 1959), war nur mit einem einzigen Exemplar am Untersuchungsort im September 1973 zu finden. 1975 jedoch

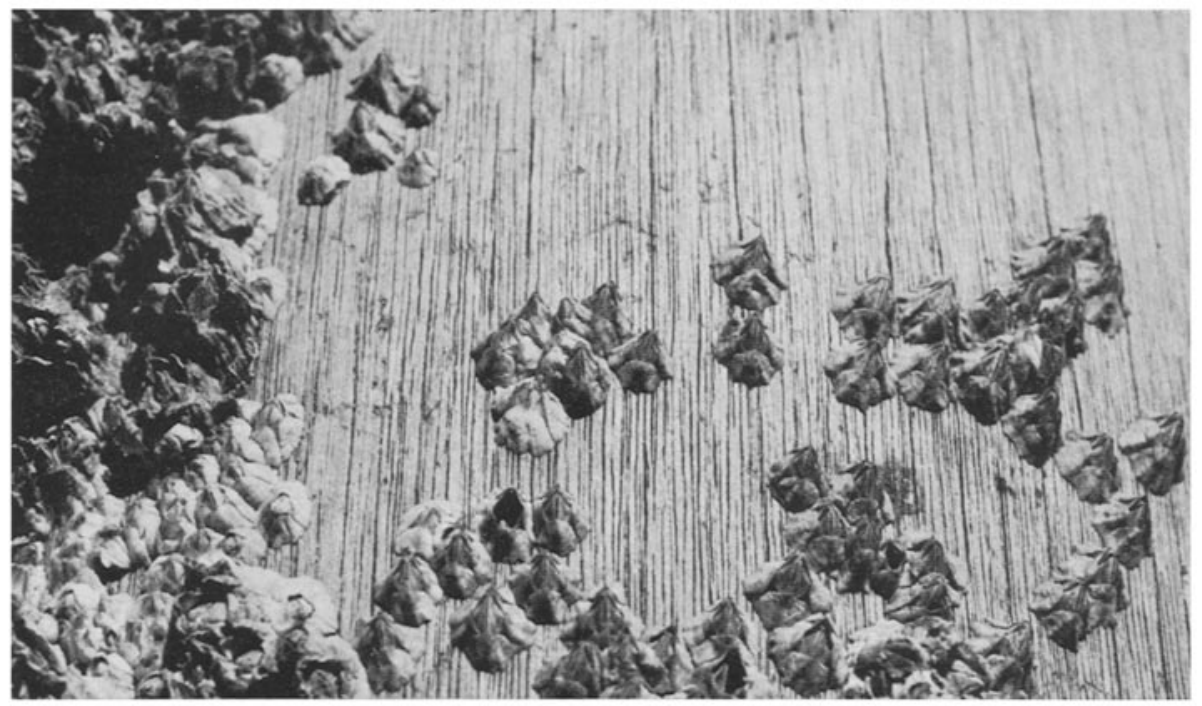

Abb. 11: Balanus balanoides und Elminius modestus im oberen Eulitoral während des Sommers 1975. Links B. balanoides (6plattig), zweijährige Tiere dunkel gefärbt, einjährige Jungtiere weiß. Rechts E. modestus (4plattig), Farbe grau

nach einem extrem milden Winter (mittlere Wassertemperatur $3,1^{\circ} \mathrm{C}$ im Februar, dem kältesten Monat) und einem extrem warmen Sommer (mittlere Wassertemperatur $20^{\circ} \mathrm{C}$ im August) trat er an den Brückenpfeilern zwischen lückenhaften Siedlungen von $B$. balanoides in großer Zahl auf (Abb. 11). Im Wattenmeer setzte er sich auf MytilusSchalen an. Dem Eisgang Anfang 1976 hielt Elminius modestus jedoch nicht stand. Er verschwand am Fundort bis auf wenige Exemplare.

\section{ZUSAMMENFASSUNG}

1. Um die Wechselbeziehung zwischen Substrat und Bewuchs zu untersuchen, wurden natürlich gewachsene Gesteine im Norden der Insel Sylt neben dem Hafen von List 
am Anleger der dänischen Autofähre angebracht und in den Jahren 1971 bis 1973 regelmäßig kontrolliert.

2. Abiotische Faktoren (Position, Wassertemperatur) und biotische Faktoren (Planktongehalt, Konkurrenten, Weidegänger und Räuber) beeinflußten die Entwicklung des Bewuchses.

3. Die Wechselbeziehungen zwischen Plankton- und Bewuchsorganismen sowie der Wassertemperaturen spiegelten sich während der Jahre 1971 und 1972 in quantitativen und qualitativen Populationsunterschieden wider.

4. Nach einer Frostperiode im März 1971 war das Plankton individuenärmer als 1972. Die Besiedlung von Balanus balanoides setzte später und weniger dicht als im folgenden Jahr nach einem milden Winter ein. Nachdem sich im Mai 1971 das Wasser des Wattenmeeres in wenigen Tagen auf $14^{\circ} \mathrm{C}$ erwärmte, entwickelten sich einige meroplanktische Formen fast explosionsartig. Es folgte auf den Substraten eine intensive Besiedlung von Mytilus edulis, Polydora ciliata und B. improvisus, die 1972 in dieser Höhe nicht mehr exreicht wurde.

5. 1972 herrschten im Gegensatz zum Vorjahr Copepoden und wärmeliebende Formen, Noctiluca miliaris, Oikopleura dioica, im Plankton vor. Unter den Bewuchsorganismen traten die Tierarten Tubularia larynx, Laomedea flexuosa und Lanice conchilega häufiger als 1971 auf.

6. Im extrem warmen Sommer 1975 (mittlere Wassertemperatur im August $20^{\circ} \mathrm{C}$ ) siedelte sich Elminizus modestus zahlreich zwischen Balanus balanoides im oberen Eulitoral an. Der von Australien eingeschleppte Balanide war bisher im Untersuchungsgebiet nur gelegentlich gefunden worden.

7. Neben dem Planktongehalt konnten als biotische Faktoren der Einfluß von Konkurrenten, Weidegängern und Räubern erfaßt werden. Konkurrenzen zwischen Balaniden einer oder nahe verwandter Arten regulierten die Abundanz. Nacktschnecken und Pantopoden beeinträchtigten als Weidegänger die Entwicklung von Hydrozoenkolonien, und der Seestern Asterias rubens bestimmte durch seine räuberische Tätigkeit die untere Verbreitungsgrenze von Mytilus edulis und den endemischen Balanidenarten. Die Grenze reichte knapp unter die Mitteltiden-Niedrigwasserlinie.

Danksagungen. Für die Anregung zu dieser Arbeit danke ich Herrn Professor Dr. Dr. h.c. A. Remane und Herrn Professor Dr. O. Kinne für die Unterstützung bei der Durchführung sowie für den Arbeitsplatz an der Litoralstation der Biologischen Anstalt Helgoland in List. Mein besonderer Dank gilt Herrn Konsul A. Lindinger, Er stellte für die In-situ-Untersuchungen einen geeigneten Standort an der dänischen Autofähre neben dem Hafen List zur Verfügung. Die Firmen H. HEMM, Natursteinwerk in Kirchheim bei Würzburg, Honst \& Jüssen und K. Rüber, Basaltwerke in Mayen/Rhld., G. Hotrtes, Granit- und Syenitwerk in Rodau/Odw. und der Solenhofener AKIIEN-Verein, Jurakalk-Steinwerke in Solnhofen lieferten und bearbeiteten das Material unentgeltlich. Für die Herstellung der Quarzsandsubstrate und die Bestimmung der technischen Daten der Natursteine danke ich Herrn Dr. G. ScrönsRunn, Materialprüfungsamt der Technischen Hochschule Darmstadt, für die Anfertigung der Versuchsrahmen Hern Dr. G. SCHEER und Herrn O. ZIERIS, Zoologische Abteilung des Darmstädter Landesmuseums. Herrn A. GREve, Wetterstation List, verdanke ich die meteorologischen Daten, Hern H. HeNnns vom Wasser- und Schiffahrtsamt Tönning die Mitteilung der Pegelstände. Weiterhin danke ich für vielfältige Unterstïtzung Hern Professor Dr. Dr. h. c. W. E. ANkel, Justus-Liebig-Universität in Gießen, Herm Dr. H. AurIcH, BAH 
List, Herrn Professor Dr. K. FaHLbusch, Technische Hochschule in Darmstadt, Herrn Professor Dr. M. Gillbricht, BAH Hamburg, Herrn Dr. H. Kesseler, BAH List, Herrn Dr. P. Kornmann, BAH Helgoland, Herrn Schiffsführer A. KRuse, BAH List, Herrn Professor Dr. E. Kullmann, Christian-Albrechts-Universität in Kiel, Herrn P. H. SAfling, BAH Helgoland, Herrn Professor Dr. W. Sch.̈̈Fer, Senckenberg-Museum in Frankfurt, Herrn M. Söhl, BAH List, Herrn K. Treutner, BAH Helgoland, Herrn Dr. E. Ziegrelmerer, BAH List.

\section{ZITIERTE LITERATUR}

BOALCH, G. T., 1957. Marine algal zonation and substratum in Beer Bay South. East-Devon. J. mar, biol. Ass. U. K. 36, 519-528.

Connele, J. H., 1964. The influence of interspecific competition and other factors on the distribution of the barnacle Chthamalus stellatus. Ecology 42, 710-723.

- 1972. Community interactions of marine rodky intertidal shores. Ann. Rev. Ecol. System. 31, 169-192.

Crisp, D. J. \& Southward, A. J., 1958. Distribution of intertidal organisms. J. mar. biol. Ass. U. K. 37, 157-208.

CROWELL, S., 1953. The regression-replacement circle of hydrants of Obelia and Campanularia. Physiol. Zoöl. 26, 319-327.

Dotr, M. S., 1967. Pioneer intertidal population and the related general vertical distribution of marine algae in Hawaii, Blumea 15, 95-105.

European Productrvity Agency, 1961. Hydrological and biological conditions in testing stations in Europe. O.E.E.C. Publications, Paris, 78 pp.

FosTER, B. A., 1971. On the determinants of the upper limit of intertidal distribution of barnacles (Crustacea, Cirripedia). J. Anim. Ecol. 40, 33-48.

Gessner, F., 1957. Meer und Strand. VEB Dt. Verl. Wiss., Berlin, 426 pp.

GOEDECKE, E., 1956b. Uber das Verhalten des Oberflächensalzgehaltes in der Deutschen Bucht während der Jahre 1873 bis 1944 in Verbindung mit langjährigen Salzgehaltsreihen der südlichen Nordsee. Ber. dt. wiss. Kommn Meeresforsch. 14, 109-146.

Gutmann, W. F., 1960. Funktionelle Morphologie von Balanus balanoides. Abh. senckenb. naturforsch. Ges. 500, 1-43.

HANCOCK, D. A., 1960. The feeding behaviour of starfish on Essex oysterbeds. J, mar. biol. Ass. U. K. 34, 313-331.

HARTOG, C. DEN, 1959a. The epilithic algal communities occurring along the coast of the Netherlands. Wentia 1, 1-271.

- 1959b. Die Seepocke Elminius modestus auf Helgoland. Beaufortia 7, 207-209.

HICKEL, W., 1975. The mesozooplankton in the wadden sea of Sylt (North-Sea). Helgoländer wiss. Meeresunters. 27, 254-262.

Hoffmann, H., 1936. Opistobranchia. Tierwelt Nord-Ostsee 9, 1-66.

Hofker, J., 1936. Die Turbellarien der Zuidersee. In: Faunistische Beobachtungen in der Zuidersee während der Trockenlegung. Z. Morph. Okol. Tiere 18, 200-216.

Johnstone, J., Scott, A., Chadwick, H. C. \& Herdman, W. A. Sir, 1925. Marine plankton. Univ. Press, Liverpool, 1-51.

Kessier, M., 1963. Die Entwicklung yon Lanice conchilega (Patras) mit besonderer Berücksichtigung der Lebensweise. Helgoländer wiss. Meeresunters. 8, 425-476.

KüHL, H., 1954. Das Auftreten von Elminius modestus DaRvin in der Elbmündung. Helgoländer wiss. Meeresunters. 5, 53-56.

LAwson, G. W. \& Norton, T. A., 1971. Some observations on littoral and sublittoral zonation at Teneriffe (Canary Isles). Botanica mar. 14, 116-120.

Linke, O., 1939. Die Biota des Jadebusenwattes. Helgoländer wiss. Meeresunters. 1, 201-348.

MEDOWs, P. S., 1969. Settlement, growth and competition in sublittoral populations of barnacles. Hydrobiologia 33, 65-92.

Mund, I., 1972. On the chemical composition, distribution and ecology of some common benthic marine algae from Iceland. Botanica mar. 15, 1-45. 
NEweLL, R. C., 1970. Biology of intertidal animals. Logos Press, London, 555 pp.

Nienburg, W., 1925. Die Algenbesiedlung des Felsstrandes und der Klippen von Helgoland, Teil II: Die Algen. Wiss. Meeresunters. (Helgoland) 15 (19), 3-15.

Nienhuis, P. H., 1969. The significance of the subtratum for intertidal algal growth on the artificial rocky shore of the Netherlands. Int. Revue ges. Hydrobiol. 54 (2). 207-215.

PAINE, R. T., 1974. Intertidal community structure. Experimental studies on the relationship between a dominant competitor and its principal predator. Oecologia 15, 93-120.

Pyeninch, K. A. \& Downing, F. S., 1949. Notes on the general biology of Tubulatia larynx. J. mar. biol. Ass. U. K. 28, 21-43.

Remane, A., 1940. Einführung in die zoologische Okologie der Nord- und Ostsee. Tierwelt Nord-Ostsee 1a, 1-238.

SOUTHWARD, A. J., 1957. On the behaviour of barnacles. III. Further observation on the influence of temperatur and age on cirral activity. J. mar. biol. Ass. U. K. 36, 323-334.

STEPHENSON, W., 1961. Experimental studies on the ecology of intertidal environments at Heron Island. Aust. J. mar. Freshw. Res. 12, 164-176.

Thorson, G., 1946. Reproduction and larval development of Danish marine bottom invertebrates. Meddr Kommn Danm. Fisk.-og Havunders (Plankton) 4, 1-523.

WERNER, B., 1958. Die Verbreitung und das jahreszeitliche Auftreten der Anthomeduse Rathkea octopunctata M. SARS, sowie die Temperaturabhängigkeit ihrer Entwicklung und Fortpflanzung. Helgoländer wiss. Meeresunters. 6, 137-170.

ZoBEzL, C. E., 1972. Bacteria, fungi and blue-green algae. In: Marine ecology. Ed. by O. KINNE. Wiley-Interscience, London, 1 (3), 1251-1270.

Anschrift der Autorin: Dr. Gertraud Luther.

Biologische Anstalt Helgoland (Litoralstation)

Hafenstraße 3

D-2282 List/Sylt

Bundesrepublik Deutschland 